\title{
Nutritional Status and Clinical Outcomes of Cardiac Patients in Acute Settings
}

\author{
Simona Cernea ${ }^{1,2}$ \\ ${ }^{1}$ Department M3/Internal Medicine IV, University of Medicine and Pharmacy, Tîrgu Mureş, Romania \\ ${ }^{2}$ Diabetes, Nutrition and Metabolic Diseases Outpatient Unit, Emergency County Clinical Hospital, Tîrgu Mureş, Romania
}

It had been extensively shown that diets and overall nutritional status are important factors influencing cardiovascular risk factors (CVRF) and outcomes. A balanced diet provides all necessary macro- and micronutrients for a healthy body composition and weight, and for promoting overall and cardiovascular health. ${ }^{1}$ Dietary intake has been long shown to significantly affect cardiovascular morbidity and mortality via complex interactions. ${ }^{2,3}$ Many observational and interventional studies have identified various nutrients that are either deleterious (such as saturated or trans- fatty acids, cholesterol) or protective (such as omega-3 polyunsaturated fatty acids, antioxidants, vitamin B12) against coronary artery disease (CAD) through changes in CVRF, and based on such findings, dietary recommendations have been made for primary and secondary CAD prevention. ${ }^{1-4}$ Traditionally, however, these recommendations have been mainly focused on individual nutrients, although beneficial dietary patterns (such as Dietary Approaches to Stop Hypertension (DASH) or Mediterranean diets) have been also advocated. ${ }^{1,4}$ Current dietary guidelines follow a dietary pattern and food-based approach and emphasize the need to adopt an overall healthy diet, because in fact the interrelationships, quantities, and combinations of nutrients in whole foods and diets are decisive for cardiovascular and general health. ${ }^{5}$

Inadequate diets with insufficient quantities and usually improper qualities of nutrients can lead to malnutrition, which in turn increases susceptibility to various acute and chronic diseases, including cardiovascular and metabolic diseases, osteoporosis, prolongs convalescence and hospital stay, and increases the risk of mortality. ${ }^{6}$ Malnutrition is especially problematic in hospitalized patients, mainly in acute settings, as it has been demonstrated to be associated with serious adverse outcomes such as alterations of immune functions with higher infection rates, muscle loss, impaired wound healing, worse response to therapy, longer and/or recurrent hospitalizations, and increased mortality rates. ${ }^{7}$ Moreover, it has been observed that even in developed countries about one third of patients admitted to the hospital are malnourished., ${ }^{7,8}$ If malnutrition is unrecognized and untreated (which apparently occurs quite often), many of these patients will experience a further decline of their nutritional status, with negative impact on recovery and other clinical outcomes. ${ }^{8}$ Therefore, a great deal of effort has been recently directed towards increasing the awareness and detection of malnutrition in hospital settings and towards the development of new, computerized tools and screening algorithms for this purpose. $^{8,9}$

In particular, cardiac patients with congestive heart failure or chronic heart disease seem to be especially vulnerable to malnutrition, which associates with negative hemodynamic outcomes, angiographic severity of coronary atherosclerosis, higher readmission rates, cardiac and all-cause death. ${ }^{10-13}$ The mechanisms are complex and insufficiently elucidated, but inflammation, impaired iron status, impaired calcium homeostasis, increased oxidative stress, cardiac cachexia/myocardial atrophy, arrhythmias, changes in blood volumes and sympathetic activity, in- 
creased coagulability/aggregation etc. play significant roles. ${ }^{10,14-16}$

In the absence of standardized assessment methods/ tools for evaluation of nutritional status of acutely hospitalized cardiac patients, various indexes have been used, which were able to predict clinical outcomes in such vulnerable patients. In this issue of the Journal of Cardiovascular Emergencies, Rus V. and colleagues have employed two practical and easy-to-use indexes for evaluation of nutritional status of cardiovascular patients admitted to an Intensive Cardiac Care Unit for acute ST (STEMI) and non-ST elevation (NSTEMI) myocardial infarction. ${ }^{17}$ The authors have used the Controlling Nutritional Status (CONUT) and Prognostic Nutritional Index (PNI) scores in 56 consecutive patients and divided them in two groups: normal to mildly impaired nutritional status (CONUT score $<3$ ) and moderate to severely impaired nutritional status (CONUT score $>3$ ), respectively. ${ }^{17}$ There were no significant differences between the two groups with regards to major CVRF, including age, smoking status, previous diagnosis of diabetes mellitus, hypertension, dyslipidemia, obesity, or previous angina pectoris/myocardial infarction. ${ }^{17}$ Notably, there was no significant difference between the body mass indexes of the two groups, their median values being in fact in the overweight range, confirming that additional nutritional evaluation is imperative for these patients. Patients with CONUT score $>3$, however, had lower total cholesterol, hematocrit, and hemoglobin values, as well as higher mean platelet volume and platelet/lymphocyte ratio, possibly indicating increased thrombogenicity. ${ }^{17}$ The authors nicely demonstrated that cardiac patients with moderate to severe nutritional impairment were more likely to present hemodynamic instability and to require inotropic medication, and had a longer intensive care and overall hospital stay than patients with normal to mild nutritional impairment. ${ }^{17}$ Although the study included a small number of subjects, the authors were able to show significant correlation of nutritional status with clinical outcomes (rate of in-hospital cardiac complications and observation time).

Certainly, this research raised further questions, and by extending the number of patients, these could possibly be addressed. Firstly, it would be interesting to observe if there is any difference regarding the nutritional status of patients with STEMI vs. NSTEMI myocardial infarction, if there is any correlation of the nutritional status with the severity/ extension of the myocardial injury and identify predictive parameters for outcomes. Secondly, it would be very useful to add other nutritional evaluation tools, such as basic anthropometric measurements (skinfolds thickness, change of weight, circumferences), dietary and nutritional determinants (protein, nitrogen, caloric and fluid balances, resting energy expenditure) and other laboratory parameters (electrolytes, serum transferrin, total iron binding capacity, creatinine to height index etc.) in order to obtain a more extensive and accurate evaluation of the nutritional status in these patients (muscle mass, subcutaneous/visceral adipose tissue, fluid etc.). Finally, it is intuitive that further examination of the inflammatory status (i.e., measurement of high-sensitivity C-reactive protein, tumor necrosis factor alpha, interleukins) and the pro-fibrotic status (cytokines, growth factors) would help to better differentiate patients with certain degrees of malnutrition at higher risk of in-hospital cardiac complications.

Although nutritional status is often overlooked in acute care settings, it has become apparent that it is a serious health issue with important medical and economic consequences, especially for cardiac patients. Despite a growing literature, malnutrition continues to be unrecognized/ undiagnosed and therefore, untreated. ${ }^{18}$ Thus, a routinely implemented structured program that aims at a formal, thorough evaluation of the nutritional status on hospital admission is of critical importance, as it may identify more fragile patients, at higher risk of complications, with therapeutic challenges and requiring special attention. Therefore, inclusion of a clinical nutrition specialist in the healthcare team is of utmost value.

Perhaps, also, development and use of reliable, validated computerized algorithms, tools, and applications for the assessment of the nutritional status is worthy. These could be practical systems that reduce the time and personnel burden by minimizing the input and rapidly analyzing the nutritional information, and can assist physicians in identifying and monitoring vulnerable patients at increased nutritional and clinical risk.

\section{REFERENCES}

1. Cernea S, Hâncu N, Raz I. Diet and coronary heart disease in diabetes. Acta Diabetol. 2003;40:S389-S400. doi: 10.1007/ s00592-003-0125-8.

2. Renaud S, Lanzmann-Petithory D. Coronary heart disease: dietary links and pathogenesis. Public Health Nutr. 2001;4:459-474.

3. $\mathrm{Hu} \mathrm{FB}$, Willett WC. Optimal diets for prevention of coronary heart disease. JAMA. 2002;288:2569-2578.

4. Eilat-Adar S, Sinai T, Yosefy C, Henkin Y. Nutritional recommendations for cardiovascular disease prevention. Nutrients. 2013;5:3646-3683. doi: 10.3390/nu5093646.

5. Bowen KJ, Sullivan VK, Kris-Etherton PM, Petersen KS. Nutrition and Cardiovascular Disease - an Update. Curr Atheroscler Rep. 2018;20:8. doi: 10.1007/s11883-018-0704-3. 
6. White JV, Guenter P, Jensen G, et al. Consensus statement: Academy of Nutrition and Dietetics and American Society for Parenteral and Enteral Nutrition: Characteristics recommended for the identification and documentation of adult malnutrition (undernutrition). JPEN J Parenter Enteral Nutr. 2012;36:275-283. doi: 10.1177/0148607112440285.

7. Barker LA, Gout BS, Crowe TC. Hospital malnutrition: prevalence, identification and impact on patients and the healthcare system. Int J Environ Res Public Health. 2011;8:514527. doi: 10.3390/ijerph8020514.

8. Guenter P, Jensen G, Patel V, et al. Addressing Disease-Related Malnutrition in Hospitalized Patients: A Call for a National Goal. Jt Comm J Qual Patient Saf. 2015;41:469-473.

9. Trtovac D, Lee J. The Use of Technology in Identifying Hospital Malnutrition: Scoping Review. JMIR Med Inform. 2018;6:e4. doi: 10.2196/medinform.7601.

10. Carr JG, Stevenson LW, Walden JA, Heber D. Prevalence and hemodynamic correlates of malnutrition in severe congestive heart failure secondary to ischemic or idiopathic dilated cardiomyopathy. Am J Cardiol. 1989;63:709-713.

11. Zhang C, Liu P, Xia K, et al. Association of Serum Prealbumin with Angiographic Severity in Patients with Acute Coronary Syndrome. Med Sci Monit. 2017;23:4041-4049.

12. Wada H, Dohi T, Miyauchi K, et al. Prognostic Impact of the Geriatric Nutritional Risk Index on Long-Term Outcomes in Patients Who Underwent Percutaneous Coronary
Intervention. Am J Cardiol. 2017;119:1740-1745. doi: 10.1016/j. amjcard.2017.02.051.

13. Aziz EF, Javed F, Pratap B, et al. Malnutrition as assessed by nutritional risk index is associated with worse outcome in patients admitted with acute decompensated heart failure: an ACAP-HF data analysis. Heart Int. 2011;6:e2. doi: 10.4081/ hi.2011.e2.

14. Moe SM. Calcium as a cardiovascular toxin in CKD-MBD. Bone. 2017; 100:94-99. doi: 10.1016/j.bone.2016.08.022.

15. Webb JG, Kiess MC, Chan-Yan CC. Malnutrition and the heart. CMAJ. 1986;135:753-758.

16. Rahman A, Jafry S, Jeejeebhoy K, Nagpal AD, Pisani B, Agarwala R. Malnutrition and Cachexia in Heart Failure. JPEN J Parenter Enteral Nutr. 2016;40:475-486. doi: 10.1177/0148607114566854.

17. Rus V, Opincariu D, Hodas R, Nyulas T, Benedek T. Interrelation between Altered Nutritional Status and Clinical Outcomes in Patients with Acute Myocardial Infarction Admitted in a Tertiary Intensive Cardiac Care Unit. Journal of Cardiovascular Emergencies. 2018;4:32-40. doi: 10.2478/jce2018-0005.

18. Kellett J, Kyle G, Itsiopoulos C, Naunton M, Luff N. Malnutrition: The Importance of Identification, Documentation, and Coding in the Acute Care Setting. J Nutr Metab. 2016;2016:9026098. doi: $10.1155 / 2016 / 9026098$. 